\title{
Contenido de humedad, proteínas y minerales en diez variedades de quínoa chilena cultivadas en distintas zonas geográficas
}

\section{Moisture, protein and mineral content of ten varieties of Chilean quinoa grown in different geographic zones}

\section{RESUMEN}

El consumo de quínoa (Chenopodium quínoa Willd) ha aumentado, renovando el interés en su composición y valor nutricional. El objetivo del estudio fue determinar los contenidos de humedad, cenizas, proteínas y algunos minerales ( $\mathrm{Fe}, \mathrm{Zn}$ y $\mathrm{Cu}$ ) de 10 variedades de quínoa chilena cultivadas en cuatro zonas geográficas, utilizando metodologías analíticas validadas. Las muestras $(n=10)$ de quínoa cultivada en Vallenar, Los Tilos, Hidango y Santa Rosa fueron analizadas en triplicado. Los métodos normalizados aplicados fueron: humedad; cenizas; proteínas; hierro, zinc y cobre, bajo los requisitos de ISO/IEC 17025:2017. Los datos se analizaron usando análisis de varianza para comparar variedades y zonas de cultivo. Las muestras contienen en promedio 16,6 $\mathrm{g}$ de proteínas $/ 100$ $g(14,4-17,5), 8,97 \mathrm{mg}$ de hierro/100 g (7,71-10,76), 3,38 $\mathrm{mg}$ de zinc/100 g $(2,17-5,30)$, y $0,83 \mathrm{mg}$ de cobre/100 $g(0,60-1,10)$. Las variedades cultivadas en Vallenar, Los Tilos e Hidango mostraron mayor contenido proteico que las de Santa Rosa $(p<0,05)$. Todas las variedades tienen un contenido destacado de los microminerales cobre, zinc y hierro. Los resultados aportan información relevante sobre el valor nutricional de la quínoa chilena, entregando datos para la actualización de las Tablas de Composición Química de alimentos.

Palabras clave: Composición; Minerales; Proteínas; Quínoa; Validación analítica; Variedades chilenas.

\footnotetext{
ABSTRACT

The intake of quinoa (Chenopodium quinoa Willd) has increased worldwide. Its revival has renewed interest in its composition and nutritional value. The aim of this study was to determine the contents of moisture, ash, protein, and some minerals ( $\mathrm{Fe}, \mathrm{Zn}$ and $\mathrm{Cu}$ ) of ten varieties of Chilean quinoa grown in various geographical zones, using validated analytical methods under rule ISO/IEC 17025:2017. Grains grown in Vallenar, Los Tilos, Hidango, and Santa Rosa were analyzed in triplicates, using previously validated analytical methodologies and certified reference materials. The normalized methods used were: moisture; ash; protein; mineral, under the ISO/IEC
}

Nazar Covarrubias" , Soraya Sandoval', Javier Vera², Claudia Núñez ${ }^{2}$, Christian Alfaro ${ }^{3}$, Mariane Lutz ${ }^{1 *}$.

\begin{abstract}
1. Centro Interdisciplinario de Estudios en Salud - CIESAL, Escuela de Medicina, Facultad de Medicina, Universidad de Valparaíso, Valparaíso, Chile.

2. Subdepartamento de Metrología y Desarrollo Tecnológico, Sección Metrología Ambiental y de Alimentos, Red Nacional de Metrología, Departamento de Salud Ambiental, Instituto de Salud Pública de Chile, Santiago, Chile 3. Instituto de Investigaciones Agropecuarias - INIA, Rayentué, Rengo, Chile.
\end{abstract}

*Dirigir correspondencia: Mariane Lutz, CIESAL, Facultad de Medicina, Universidad de Valparaíso. Angamos 655, Reñaca, Viña del Mar, Chile. Mail: mariane.lutz@uv.cl

Este trabajo fue recibido el 08 de enero de 2020. Aceptado con modificaciones: 27 de abril de 2020 . Aceptado para ser publicado: 08 de mayo de 2020.

17025:2017 norm. Data were analyzed using ANOVA to compare varieties and growth zones. The analyzed quinoa grains contain a mean of $16.6 \mathrm{~g}$ proteins $/ 100 \mathrm{~g}$ (range 14.4-17.5), $8.97 \mathrm{mg}$ iron/100 g (range 7.71-10.76), $3.38 \mathrm{mg}$ zinc $/ 100 \mathrm{~g}$, and $0.83 \mathrm{mg}$ copper $/ 100 \mathrm{~g}$ (range 0.60-1.10). The varieties grown in Vallenar, Los Tilos and Hidango showed higher protein content compared to Santa Rosa $(p<0.05)$. All varieties exhibit considerable microminerals content, such as copper, zinc, and iron. These results provide relevant information about the nutritional value of Chilean quinoa and updated reliable data for Food Composition Tables.

Keywords: Analytical validation; Chilean varieties; Composition; Iron; Minerals; Protein; Quinoa. 


\section{INTRODUCCIÓN}

La quínoa o quinua (Chenopodium quínoa Willd) ha sido utilizada tradicionalmente como alimento por los pueblos andinos, formando parte de su patrimonio cultural ancestral, y ha sido cultivada desde los $2^{\circ}$ latitud Norte (Colombia) a $47^{\circ}$ latitud Sur (Chile) por siglos'. El cultivo fue domesticado por civilizaciones antiguas en América ${ }^{2}$ y fue casi extinguida por los colonos españoles ${ }^{3}$, pero en la última década ha experimentado un renacimiento ${ }^{4}$. En Chile, el ecotipo prevalente es Salares en la zona desértica del norte ${ }^{5}$, en tanto que en las zonas centro y sur predomina el ecotipo Costero, con rangos de lluvia de 500 a 1500 $\mathrm{mm}$ anuales $\mathrm{s}^{6,7}$. Debido a su capacidad de adaptarse al déficit hídrico, es un cultivo interesante en zonas áridas y semiáridas, y se está expandiendo en todo el mundo ${ }^{1,8}$.

La quínoa es altamente nutritiva. El Codex Alimentarius Internaciona ${ }^{9}$ estableció una regulación para resguardar la mínima calidad del grano, estandarizando el máximo contenido de humedad de los granos en 13,5\%, y un contenido proteico mínimo de 10,0\% para su comercialización. La mayor parte de la quínoa producida en el mundo contiene sobre $12 \%$ de proteína, en un rango promedio de 12 a $17 \mathrm{~g} / 100 \mathrm{~g}^{4}$. Además, es reconocida como buena fuente de minerales, incluyendo potasio, calcio, magnesio, hierro, fósforo, zinc, manganeso y cobre ${ }^{10,11}$. Considerando la creciente producción de quínoa en Chile, el gran interés por estandarizarla ${ }^{12}$ y la necesidad de contar con datos analíticos validados sobre su composición, tanto para caracterizar su valor nutricional como también con propósitos de optimizar su comercialización, el objetivo de este estudio fue determinar el contenido de humedad, cenizas, proteínas y minerales ( $\mathrm{Fe}, \mathrm{Zn}$ y Cu ) de diez variedades de quínoa chilena, validando previamente las metodologías analíticas oficiales a aplicar para entregar datos confiables aptos para ser incorporados en las Tablas de Composición de Alimentos Chilenos.

\section{MATERIALES Y MÉTODOS Muestras}

Se analizaron granos de Chenopodium quínoa sembrados en campos experimentales ubicados en diferentes localidades a nivel nacional, correspondientes a: Vallenar $\left(28^{\circ} 34^{\prime} \mathrm{S}\right.$,

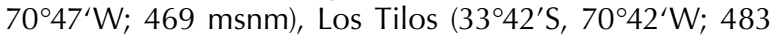
msnm), Hidango ( $\left.34^{\circ} 06^{\prime} \mathrm{S}, 71^{\circ} 47^{\prime} \mathrm{W}, 269 \mathrm{msnm}\right)$, y Santa Rosa (36 $\left.31^{\prime} \mathrm{S}, 71^{\circ} 54^{\prime} \mathrm{W}, 220 \mathrm{msnm}\right)$. Las variedades analizadas en cada localidad fueron: AZ-1, AZ-2, AZ-3 (ecotipos de Zonas Áridas); Morada Hidango, Verde Hidango, Chen 46 27C, Cahuil, EAM 62-A, Roja INIA, y Regalona-B (hibridación genética), las que se muestran en la figura 1 . En el campo experimental se sembraron con un diseño de 10 bloques aleatorizados con cuatro filas de $4 \mathrm{~m} \times 2 \mathrm{~m}$, separadas por 0,5 $\mathrm{m}$, en un área total de $8 \mathrm{~m}^{2}$. En Vallenar (Región de Atacama), Los Tilos (Región Metropolitana) y Santa Rosa (Región del Biobío) los ensayos de cultivos fueron regados, mientras Hidango (Región del Libertador Bernardo O'Higgins), que es una localidad representativa del Secano Central, solo tuvo los aportes hídricos de la lluvia en la temporada de cultivo.

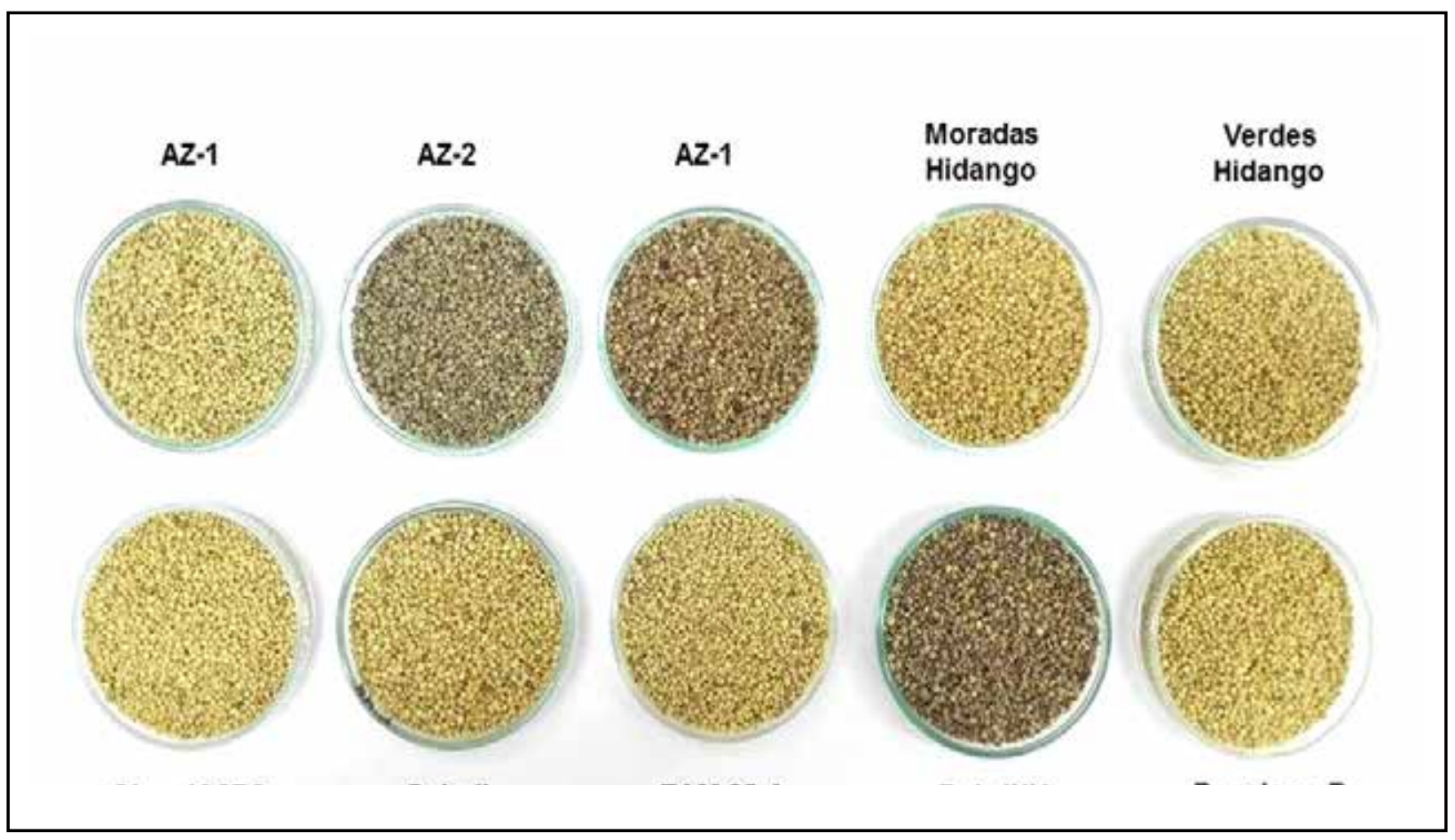

Figura 1: Variedades de granos de quínoa analizadas. 
La cosecha se realizó en 2017, y las muestras de quínoa en grano fueron recolectadas e identificadas individualmente por el INIA y enviadas al Instituto de Salud Pública (ISP), en Santiago, donde fueron almacenadas protegidas de la luz y humedad. Posteriormente, los granos se molieron en molino Fritsch ${ }^{\circledR}$ Pulverisette 14 a 8000 rpm durante $20 \mathrm{~s}$ para evitar su calentamiento y se tamizaron a $1,0 \mathrm{~mm}$. El tratamiento se realizó en condiciones ambientales de 22 a $25^{\circ} \mathrm{C}$ y 30 a $50 \%$ humedad relativa. Las muestras molidas se mantuvieron en bolsas herméticas al vacío para evitar la humedad y contaminación hasta su análisis.

\section{Métodos de Análisis}

La composición química de las 10 variedades de quínoa se determinó utilizando métodos de ensayo oficiales que fueron validados analíticamente a través de materiales de referencia certificados de grano trazables al Sistema Internacional de Unidades, para determinar su precisión y confiabilidad analítica. El aseguramiento de la calidad de las mediciones y validaciones de métodos para medir las características nutricionales fue parte del proyecto Quality assurance and metrological traceabily of analytical parameters used for the characterization of quinoa, que proporcionó un patrón de quínoa con certificación dada por los respectivos Institutos Nacionales de Metrología de la Región Latinoamericana para medir las características nutricionales de la quínoa que cumple con ISO 17034:2016 e ISO Guide 35:2017. Las metodologías aplicadas fueron:

- Humedad: método gravimétrico primario ISO 712:2009 (Cereales y derivados - Determinación del contenido de humedad), basado en la determinación gravimétrica por pérdida de masa de la muestra desecada hasta masa constante en estufa. Se utilizó estufa por aire por convección Labtech ${ }^{\circledast}$ Lib-080M monitorizada por termocupla $\mathrm{VWR}^{\circledR} \mathrm{AV}$ trazable al SI. Las muestras fueron masadas en balanza analítica Sartorius ${ }^{\circledR}$ modelo LA 230 S trazable al SI.

- Proteínas: método primario titrimétrico ISO 1871:2009 (Food and feed products - General guidelines for the determination of nitrogen by the Kjeldahl method), usando como factor de conversión de nitrógeno $(\mathrm{N})$ a proteínas 6,25, como recomienda el Método Oficial del Codex Alimentario ${ }^{13}$. Las muestras fueron digeridas en el equipo Kjeldatherm KB/KBL Gerhardt ${ }^{\circledR}-\mathrm{KB} 8 \mathrm{~S}$ y destiladas en el equipo Gerhardt ${ }^{\circledR}$-Vadopest 300, ambos certificados (ISO 712 para humedad e ISO 1871) para proteínas según Codex Alimentarius International en 2018. Para asegurar la calidad y trazabilidad del ensayo se utilizó materiales de referencia certificados (MRC) EMR ${ }^{\circledR}-\mathrm{BC} 382$ (0614) harina de trigo para $\mathrm{N}$ $(1,851 \pm 0,017 \mathrm{~g} / 100 \mathrm{~g}) ;$ MRC $\mathrm{EMR}^{\circledR}$-BC381 (0209) harina de centeno para $\mathrm{N}(1,562 \pm 0,1 \mathrm{~g} / 100 \mathrm{~g})$.

- Cenizas: método gravimétrico primario ISO 2171:2007 (Cereales, legumbres y derivados - Determinación de residuos de incineración). Las muestras fueron calcinadas en mufla Nabertherm ${ }^{\circledR}$ modelo NH7. Para asegurar la calidad se utilizó MRC ERM ${ }^{\circledR}$-BC382 harina de centeno para dar trazabilidad a la medición.

- Minerales: método secundario de espectrofotometría de absorción atómica (EAA) NMKL 161:1998 (Metals. Determination by atomic absorption spectrophotometry after wet digestion in a microwave oven). Se realizó una digestión ácida en horno microondas Anton Paar ${ }^{\circledR}$ Multiwave PRO y posterior cuantificación en un equipo PerkinElmer ${ }^{\circledR}$ - PinAAcle 900T. En la cuantificación de metales específicos se utilizó MRC de Fe: estándar NIST SRM ${ }^{\circledR}$ 3168a $(10007 \pm 20 \mathrm{mg} / \mathrm{kg})$; Cu: estándar NIST certificado de cobre SRM ${ }^{\circledR} 3114(10005 \pm 24 \mathrm{mg} /$ $\mathrm{kg})$.

Para asegurar la validez de los resultados, las muestras fueron analizadas en triplicado o duplicado y en paralelo a un material de referencia certificado, realizándose re-análisis de muestras según el caso.

- Estadística: para cada nutriente se determinó la incertidumbre de medición (con un intervalo de confianza del 95\%, es decir, k=2) metrológicamente trazable, a través de una cadena ininterrumpida de calibraciones documentadas hasta una referencia, mediante procedimientos de medición que permitieran relacionar cada resultado con el Sistema Internacional de unidades. Para ello, se analizaron 10 muestras de cada variedad de quínoa de cada una de las 4 zonas de cultivo, todas por triplicado, totalizando 120 muestras para cada elemento medido. Se realizó un análisis de varianza (ANOVA) de un factor, utilizando el paquete "análisis de datos" de Excel para determinar las diferencias significativas entre las variedades y zonas geográficas de quínoa chilena. Las diferencias se calcularon con un nivel de significación de $\alpha=0,05$ y un intervalo de confianza del 95\% $(p<0,05)$.

\section{RESULTADOS}

El tenor de humedad de las diez variedades de quínoa chilena analizadas fue similar, con valores en un rango de 8,8 $\pm 0,3 \mathrm{~g} / 100 \mathrm{~g}$ en Verde Hidango a 9,3 $\pm 0,4 \mathrm{~g} / 100 \mathrm{~g}$ en AZ-2. Sin embargo, se observaron diferencias según zona de cultivo: la humedad de los granos fue mayor en aquellos provenientes de Vallenar $(9,3 \pm 0,2 \mathrm{~g} / 100 \mathrm{~g})$ y Santa Rosa $(9,2 \pm 0,2 \mathrm{~g} / 100 \mathrm{~g})$, ambos mayores $(\mathrm{p}<0,05)$ a los obtenidos en las muestras provenientes de Los Tilos $(8,9 \pm 0,2 \mathrm{~g} / 100 \mathrm{~g})$ e Hidango $(9,0 \pm 0,1 \mathrm{~g} / 100 \mathrm{~g})$.

El contenido de proteínas de las 10 variedades de quínoa se presenta en la tabla 1 . El promedio fue de $16,6 \mathrm{~g} / 100 \mathrm{~g}$. El contenido medio fue significativamente más alto en los granos de quínoa cultivada en Vallenar, Hidango y Los Tilos $(17,5 \pm 0,7,17,5 \pm 0,5$ y $16,8 \pm 0,6$ $\mathrm{g} / 100 \mathrm{~g}$, respectivamente), respecto a los de Santa Rosa $(14,5 \pm 0,6 \mathrm{~g} / 100 \mathrm{~g})(\mathrm{p}<0,05)$. El tenor más alto de proteínas se observó en la variedad AZ-1 proveniente de Vallenar $(19,0 \pm 0,1 \mathrm{~g} / 100 \mathrm{~g}), 44 \%$ mayor al más bajo, observado en AZ-2 de Santa Rosa $(13,1 \pm 0,1 \mathrm{~g} / 100 \mathrm{~g})$. 
Tabla 1. Contenido de proteína (g/100 g) en muestras de quínoa según variedad y origen.

\begin{tabular}{|c|c|c|c|c|c|c|c|}
\hline \multirow[b]{2}{*}{ Variedad } & \multicolumn{5}{|c|}{ Origen } & \multirow[b]{2}{*}{ DE } & \multirow[b]{2}{*}{$\% C V$} \\
\hline & $\begin{array}{l}\text { Vallenar } \\
\quad(n=2)\end{array}$ & $\begin{array}{l}\text { Los Tilos } \\
(n=2)\end{array}$ & $\begin{array}{l}\text { Hidango } \\
(n=2)\end{array}$ & $\begin{array}{c}\text { S. Rosa } \\
(n=2)\end{array}$ & $\begin{array}{l}\text { Media } \\
\text { variedad }\end{array}$ & & \\
\hline $\begin{array}{l}\text { AZ-1 } \\
\text { autofecundación }\end{array}$ & $18,9 \pm 0,1$ & $18,3 \pm 0,1$ & $18,2 \pm 0,3$ & $15,3 \pm 0,1$ & $17,7 \pm 1,7$ & 1,6 & 9,2 \\
\hline $\begin{array}{l}\text { AZ } 2 \\
\text { autofecundación }\end{array}$ & $15,7 \pm 0,1$ & $15,6 \pm 0,1$ & $16,5 \pm 0,0$ & $13,1 \pm 0,1$ & $15,3 \pm 1,5$ & 1,5 & 9,7 \\
\hline $\begin{array}{l}\text { AZ } 3 \\
\text { autofecundación }\end{array}$ & $17,5 \pm 0,1$ & $15,9 \pm 0,1$ & $17,3 \pm 0,1$ & $13,8 \pm 0,1$ & $16,1 \pm 1,7$ & 1,7 & 10,5 \\
\hline $\begin{array}{l}\text { Moradas Hid. } \\
\text { Selección masal }\end{array}$ & $17,7 \pm 0,3$ & $16,5 \pm 0,1$ & $18,0 \pm 0,1$ & $13,7 \pm 0,1$ & $16,5 \pm 2,0$ & 2,0 & 11,9 \\
\hline $\begin{array}{l}\text { Verde Hidango } \\
\text { Selección masal }\end{array}$ & $17,5 \pm 0,2$ & $17,8 \pm 0,1$ & $18,2 \pm 0,1$ & $15,1 \pm 0,1$ & $17,2 \pm 1,4$ & 1,4 & 8,1 \\
\hline $\begin{array}{l}\text { Chen46 27-C } \\
\text { Selección masal }\end{array}$ & $17,6 \pm 0,0$ & $16,5 \pm 0,1$ & $17,6 \pm 0,1$ & $14,4 \pm 0,1$ & $16,5 \pm 1,5$ & 1,5 & 9,0 \\
\hline $\begin{array}{l}\text { Cahuil } \\
\text { Selección masal }\end{array}$ & $18,1 \pm 0,1$ & $17,7 \pm 0,1$ & $17,7 \pm 0,1$ & $14,6 \pm 0,2$ & $17,0 \pm 1,6$ & 1,6 & 9,5 \\
\hline $\begin{array}{l}\text { EAM 62-A } \\
\text { Selección masal }\end{array}$ & $16,7 \pm 0,1$ & $16,2 \pm 0,2$ & $16,9 \pm 0,1$ & $14,2 \pm 0,0$ & $16,0 \pm 1,2$ & 1,2 & 7,6 \\
\hline $\begin{array}{l}\text { Roja INIA } \\
\text { Selección masal }\end{array}$ & $18,0 \pm 0,1$ & $16,6 \pm 0,1$ & $17,6 \pm 0,1$ & $15,0 \pm 0,1$ & $16,8 \pm 1,4$ & 1,3 & 8,0 \\
\hline $\begin{array}{l}\text { Regalona-Baer } \\
\text { Hibridación }\end{array}$ & $17,4 \pm 0,1$ & $17,1 \pm 0,1$ & $16,8 \pm 0,0$ & $15,6 \pm 0,1$ & $16,7 \pm 0,8$ & 0,8 & 4,8 \\
\hline Media general & $17,5 \pm 0,7^{\mathrm{a}}$ & $16,8 \pm 0,6^{a}$ & $17,5 \pm 0,5^{\mathrm{a}}$ & $14,5 \pm 0,6^{b}$ & 16,6 & & \\
\hline
\end{tabular}

General guidelines for the determination of nitrogen by the Kjeldahl; ISO 1871: 2009. Trazable a NIST SRM 723 e y a IRRM ERM ${ }^{\circledR}$-BC381. Incertidumbre expandida $(U k=2)$. Los valores se presentan como $\mathrm{X} \pm \mathrm{DE}$. DE= desviación estándar; \%CV= coeficiente de variación porcentual. Distinto superíndice en el valor de la media general de cada columna indica diferencia significativa $(p<0,05)$.

El contenido de cenizas de las variedades de quínoa se presenta en la figura 2. El contenido de hierro de las 10 variedades de quínoa chilena analizadas se presenta en la figura 3. El tenor de hierro (promedio $8,97 \mathrm{mg} / 100 \mathrm{~g}$ ) es $98 \%$ superior al valor reportado por $\mathrm{FAO}^{13}$. Según la zona de cultivo, destaca el hierro de las muestras provenientes de Santa Rosa, que presentaron una alta dispersión (rango de $6,50 \pm 0,29$ a $26,36 \pm 0,31 \mathrm{mg} / 100 \mathrm{~g}$ ), debido a que las variedades AZ-1, Chen 44 27-C y EAM 62-A mostraron niveles muy altos $(p<0,05)$. Este análisis fue repetido completamente, revisando cada etapa del proceso de análisis, lo que llevó a completarlo en 9 muestras (27 lecturas) en total, en las cuales se repitieron los resultados que se informan. El valor máximo observado, en la variedad EAM 62, es 405\% mayor que el valor más bajo de la misma región (AZ-2). Asimismo, los granos contienen cantidades importantes de zinc (Figura 4), y cobre (Figura 5), especialmente aquellos provenientes de Hidango $(p<0,05)$. El zinc varió según la zona geográfica de donde se obtuvo la muestra, siendo similar en Vallenar y Santa Rosa $(p<0,05)$. El contenido medio fue más alto en los granos de Hidango $(53,0 \pm 6,7 \mathrm{mg} / \mathrm{kg})$, superando al de las demás zonas de cultivo $(p<0,05)$. El tenor de zinc en 
la variedad Verde Hidango cultivada en Hidango $(6,23 \pm$ $0,03 \mathrm{mg} / 100 \mathrm{~g}$ ) es $494 \%$ más alto que el de la variedad AZ-2 de Los Tilos $(1,26 \pm 0,02 \mathrm{mg} / 100 \mathrm{~g})$, zona en la que se observó una alta variabilidad entre las variedades, llegando a un máximo de 4,81 $\pm 0,33 \mathrm{mg} / 100 \mathrm{~g}$ (Cahuil). En cuanto al cobre, las muestras presentaron un contenido promedio mayor $(0,83 \mathrm{mg} / 100 \mathrm{~g})$ al valor informado por $\mathrm{FAO}^{13}$, de 0,59 $\mathrm{mg} / 100 \mathrm{~g}$. El contenido más alto se observó en la variedad EAM 62-A cultivada en Hidango (1,32 $\pm 0,02 \mathrm{mg} / 100 \mathrm{~g}$ ), un $281 \%$ mayor que el valor más bajo, de la variedad Chen 46 27-C cultivada en Santa Rosa $(0,47 \pm 0,02 \mathrm{mg} / 100 \mathrm{~g})$ $(p<0,05)$. En Los Tilos se observa una gran variabilidad en contenido de cobre entre variedades $(0,50 \pm 0,02$ en AZ-2 a $1,21 \pm 0,07 \mathrm{mg} / 100 \mathrm{~g}$ en Cahuil).

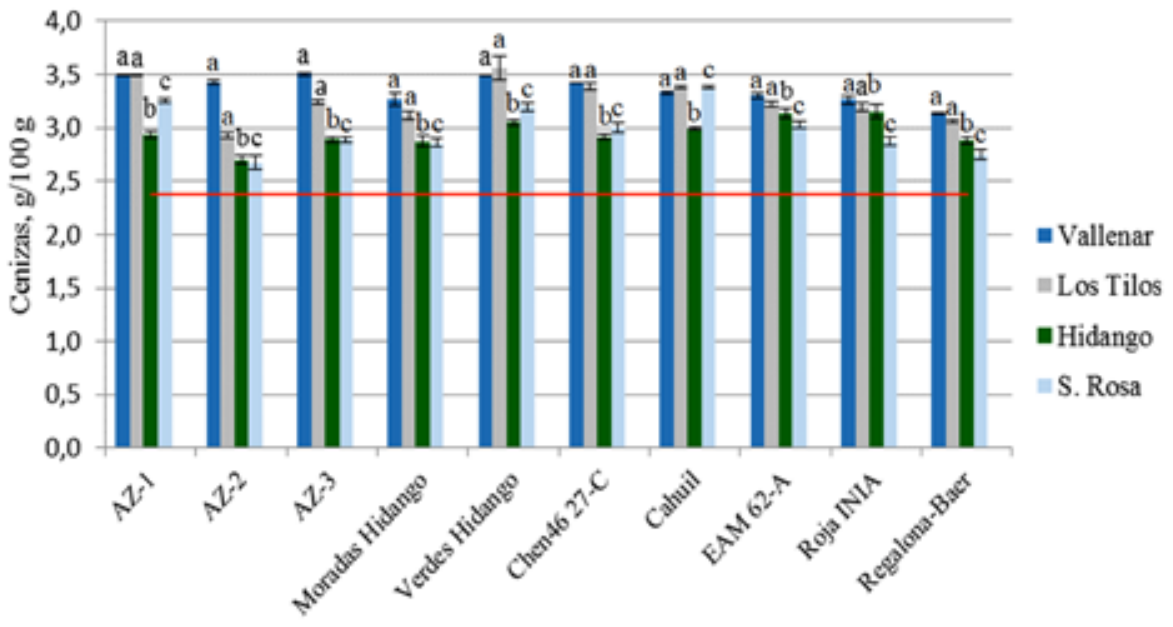

Figura 2: Contenido promedio de cenizas ( $\mathrm{g} / 100 \mathrm{~g}$ ) en quínoa chilena según variedad y origen. Los valores se expresan como $\mathrm{X} \pm \mathrm{DE}$. La línea roja muestra el valor promedio reportado por $\mathrm{FAO}^{15}$. Diferentes letras sobre las barras en cada variedad indican diferencia significativa $(\mathrm{p}<0,05)$.

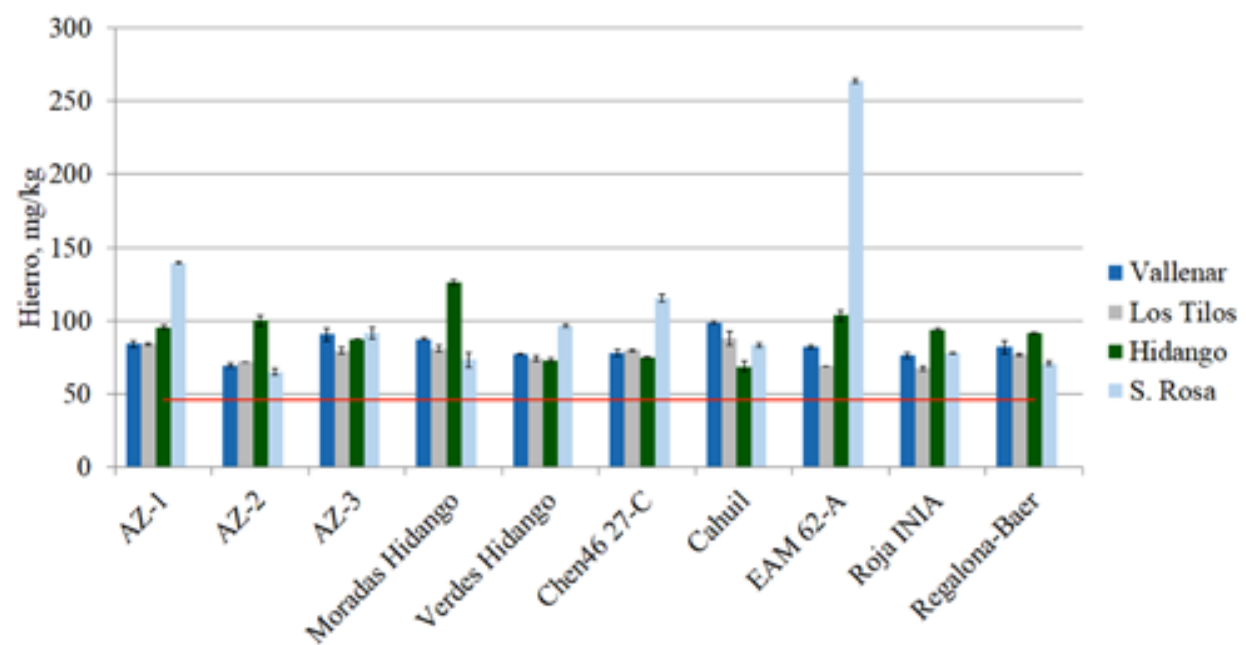

Figura 3: Contenido promedio de hierro $(\mathrm{mg} / \mathrm{kg})$ en quínoa chilena según variedad y origen. Los valores se expresan como $\mathrm{X} \pm \mathrm{DE}$. La línea roja muestra el valor promedio reportado por $\mathrm{FAO}^{15}$. Los valores promedio son semejantes entre sí. 


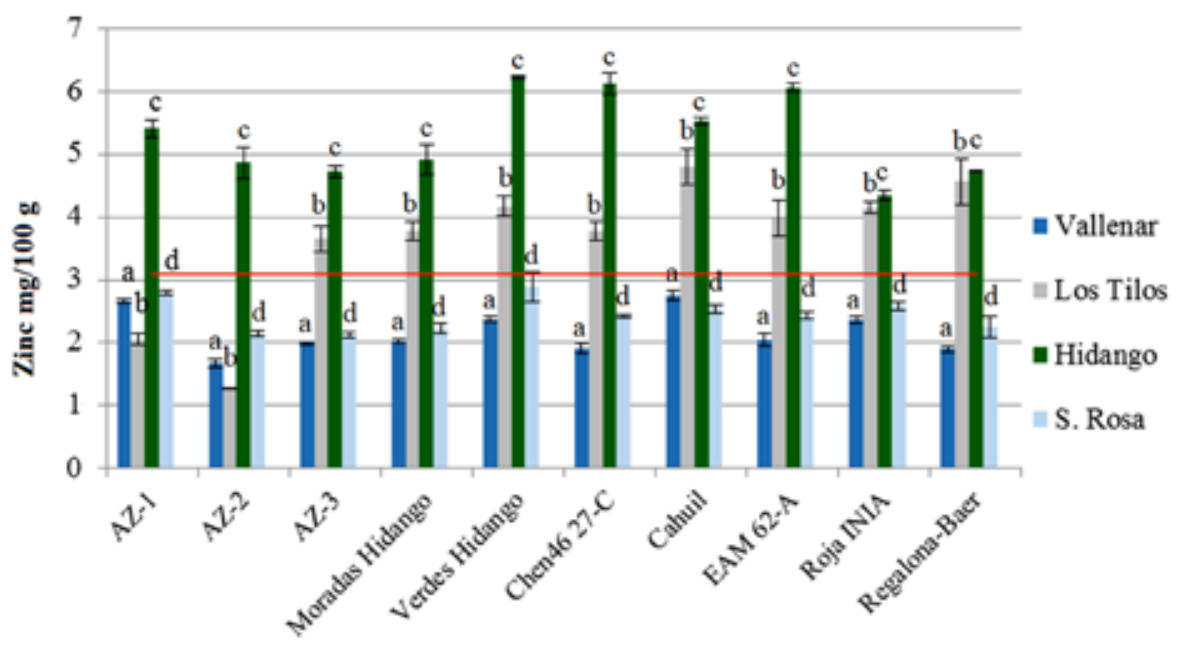

Figura 4: Contenido promedio de zinc $(\mathrm{mg} / \mathrm{kg}$ ) en quínoa chilena según variedad y origen. Los valores se expresan como $X \pm D E$. La línea roja muestra el valor promedio reportado por $F O^{15}$. Diferentes letras sobre las barras en cada variedad indican diferencia significativa según zona de cultivo $(p<0,05)$.

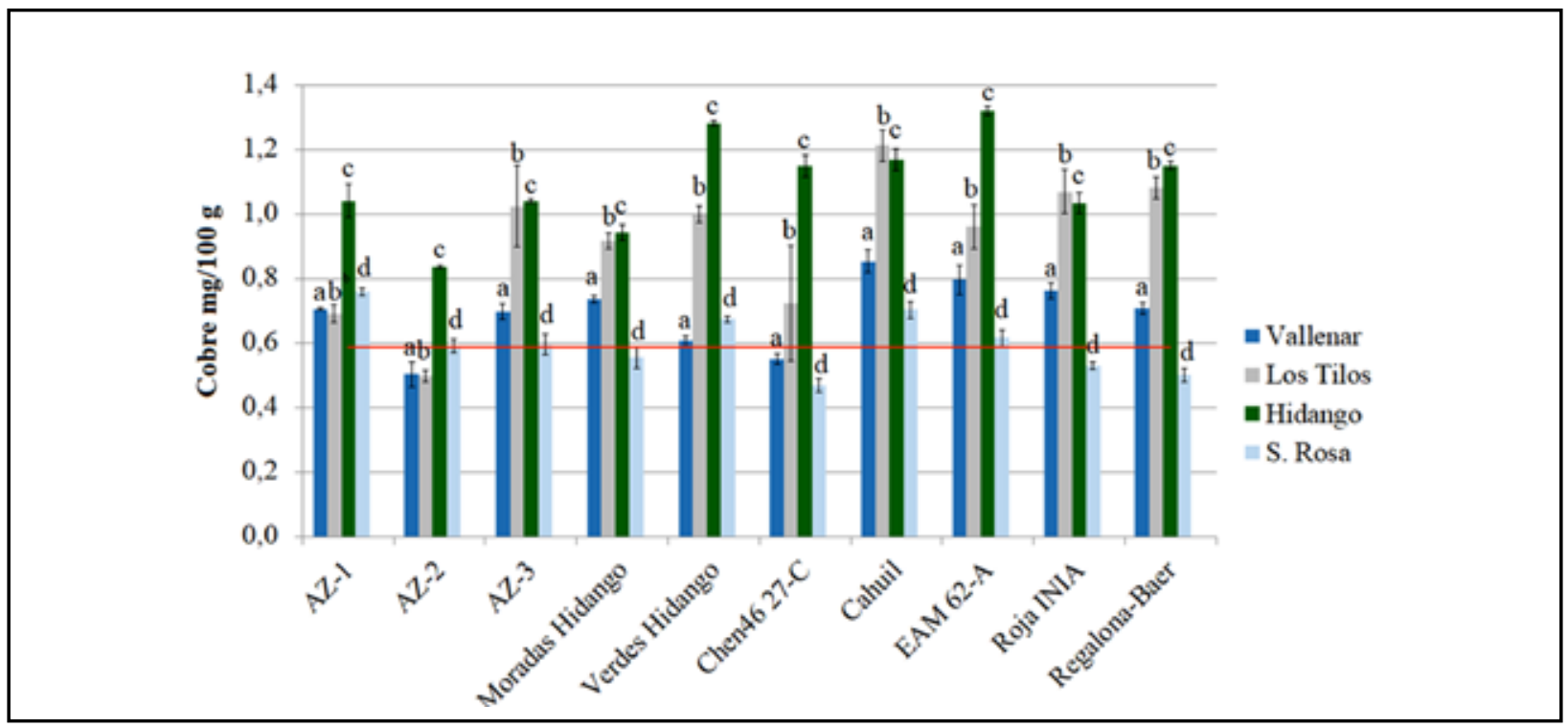

Figura 5: Contenido promedio de cobre $(\mathrm{mg} / \mathrm{kg})$ en quínoa chilena según variedad y origen. Los valores se expresan como $\mathrm{X} \pm \mathrm{DE}$. La línea roja muestra el valor promedio reportado por $\mathrm{FAO}^{15}$. Diferentes letras sobre las barras en cada variedad indican diferencia significativa según zona de cultivo $(p<0,05)$.

\section{DISCUSIÓN}

El presente trabajo muestra resultados del análisis del contenido de humedad, proteínas, cenizas, hierro, zinc y cobre de diez variedades de quínoa chilena, utilizando metodologías analíticas oficiales validadas, con materiales de referencia estandarizados para este fin. De este modo, se cumple con el objetivo de aportar información sobre la caracterización nutricional de la quínoa chilena y, del mismo modo, contribuir con datos sobre este alimento a las Tablas de Composición de Alimentos chilenos. Por 
otra parte, estudiar las variaciones de la composición de variedades de quínoa chilena y el efecto de su cultivo en diferentes zonas geográficas en condiciones estandarizadas permite su comparación, y pone en evidencia relevando las diferencias que se presentan en una variedad al ser cultivada en determinada zona.

El promedio de humedad de las muestras analizadas, de $9,1 \mathrm{~g} / 100 \mathrm{~g}$, es un $32 \%$ más bajo que el valor reportado en la Tabla de $\mathrm{FAO}^{14}$, de 13,3 g/100 g.

Los resultados de contenido proteico de las variedades de quínoa analizada ponen en evidencia que el grano chileno posee más proteína que el de otros países, como Perú, con 13,6 g/100 g ${ }^{16}$. El valor medio obtenido en este estudio es un $17 \%$ mayor al reportado en la Tabla de $\mathrm{FAO}^{14}$, correspondiente a 14,1 g/100 g. El valor medio supera a los informados previamente en otras muestras de quínoa chilena y peruana, en un rango de 11,3 a 16,1 $\mathrm{g} / 100 \mathrm{~g}^{11}$ y de 14,0 a $15,5 \mathrm{~g} / 100 \mathrm{~g}^{17}$, respectivamente. El estándar de Codex para quínoa ${ }^{15}$ establece que el mínimo contenido de proteína en este grano debe ser de 10,0 g/100 g. El rango de contenido de proteínas en quínoa ha sido descrito entre $7,5 \%$ y $22,1 \%$, con un promedio de $14,6 \%{ }^{4,18,19,20,21,22,23,24,25,26}$. Sin embargo, existe una dificultad al comparar los datos obtenidos por diferentes autores, ya que no siempre se consigna qué factor de conversión de $\mathrm{Na}$ proteínas fue aplicado, y algunos han utilizado el factor 5,7 (para cereales) en lugar del factor que actualmente se acepta para la quínoa, de 6,25. También es relevante saber si la quínoa analizada es fresca o se ha sometido a pre-tratamiento térmico: la cocción en agua hirviente, principal forma de preparación culinaria, reduce su contenido de proteínas. Así, la base de datos nutricionales del Departamento de Agricultura de los EE.UU. reporta 13,9 $\mathrm{g}$ de proteínas /100 g en granos crudos y 4,4 g/100 g en granos $\operatorname{cocidos}^{27}$.

Algunos valores reportados del contenido proteico en quínoa chilena varían según la zona geográfica, siendo menores a 12 g/100 g en la zona Central y entre 14 y 16 g/100 g en la zona Sur, lo que puede atribuirse a suelos volcánicos con mayor contenido de $\mathrm{N}^{28}$. En un estudio reciente, en quínoa Costera de la Región de Valparaíso se encontró un rango de 12,4 a 13,8 g/100 g de proteínas ${ }^{29}$. Vega-Gálvez et $\mathrm{al}^{30}$ reportaron 12 a $14 \mathrm{~g} / 100 \mathrm{~g}$ de proteínas en granos del norte, 11 a 12 g/100 g en el centro y 14 a 16 g/100 g en el sur del país. El alto tenor proteico de la quínoa chilena es un factor de calidad que debiera contribuir a mejorar su comercialización a nivel nacional e internacional ${ }^{31}$.

Todas las muestras presentaron un tenor de minerales superiores a los reportados por $\mathrm{FAO}^{14}$, sin diferencia entre sí, con una media general de 3,1 g/100 g. El valor medio fue de 3,1 g/100 g, un 32\% más alto que el reportado en la Tabla de Composición de Quínoa de $\mathrm{FAO}^{15}$, que alcanza a 2,4 g/100 g. Esto se puede atribuir, al menos en parte, al alto contenido de minerales de los suelos chilenos, especialmente en la zona salina del norte y suelos volcánicos del sur. El valor medio concuerda con el contenido de cenizas en quínoas chilenas y peruanas, con rangos de 3,2 a 3,6 g/100 $\mathrm{g}^{11}$ y 3,0 a 5,5 g/100 ${ }^{16}$, respectivamente.

Las muestras de quínoa analizadas mostraron un alto contenido de hierro, independientemente de su zona de origen. Los valores obtenidos son superiores a los reportados por Torrez et a ${ }^{32}$ en diez variedades de quínoa del altiplano boliviano ( 45,0 a $57,5 \mathrm{mg} / \mathrm{kg}$ ), y un $20 \%$ mayor con respecto a la quínoa peruana $(75,0 \mathrm{mg} / \mathrm{kg})^{16}$. No se observó diferencia significativa en el contenido de hierro de la quínoa entre zonas de cultivo, probablemente debido a la alta variabilidad entre las muestras cultivadas en la misma zona. En aquellas provenientes de Santa Rosa, el rango de concentraciones difirió aproximadamente en $200 \mathrm{mg} / \mathrm{kg}$ entre las de mayor y menor contenido, resultado que fue analíticamente validado. No se cuenta con una explicación a esta variabilidad, que podría estar asociada a una condición en algunas semillas para retener el mineral, lo cual sería necesario explorar a futuro. Los niveles observados de zinc son comparables con los reportados por Martínez et al ${ }^{11}$, de 2,73 a 5,01 mg/100 $\mathrm{g}$ en las seis variedades chilenas provenientes de tres zonas geográficas. El tenor de cobre observado es comparable al reportado en diez variedades de quínoa del altiplano boliviano $(0,63 \text { a } 1,44 \mathrm{mg} / 100 \mathrm{~g})^{29}$, como asimismo con valores observados por Martínez et $\mathrm{al}^{11}$, en un rango de 0,75 a 1,52 mg/100 g, en seis variedades de quínoa chilena de tres zonas geográficas. El contenido de los microminerales en quínoa es altamente dependiente de las condiciones del terreno de cultivo, por lo que sería de gran interés conocer su composición, así como las condiciones de riego y la pluviometría, entre otras condiciones agroclimáticas que, de ser debidamente controladas, pueden contribuir a la mejora de su aporte nutricional. Los resultados están en concordancia con el alto contenido de cenizas en las muestras analizadas, cuyo valor más alto se encontró en las variedades cultivadas en Hidango, al igual que ocurrió con los contenidos de cobre y zinc $(p<0,05)$.

\section{CONCLUSIONES}

El promedio de humedad de los granos de quínoa chilena es más bajo al reportado por FAO y diversos autores, en tanto que el de proteínas, en general, es mayor al de aquella cultivada en otros países. Además del alto tenor proteico, en las variedades chilenas destaca el contenido de los microminerales cobre, zinc y hierro. Los resultados validados de este estudio son de gran interés para la valorización de este cultivo ancestral en el país, contribuyendo a su reposicionamiento como alimento saludable y para fomentar su justa comercialización, basada en parámetros objetivos de calidad nutricional, dentro y fuera del país.

\section{BIBLIOGRAFÍA}

1. Bazile D. State of the art of quinua in the world in 2013. FAO, Santiago de Chile and CIRAD, Montpellier, France, 2014, p. 724

2. Kadereit G, Borsch T, Welsing K, Freitag H. Phylogeny of Amaranthaceae and Chenopodiaceae and the evolution of C4 photosynthesis. Int J Plant Sci. 2003; 164: 959-986. 
3. Vega-Gálvez A, Miranda $M$, Vergara J, Uribe $E$, Puente $L$, Martínez EA. Nutrition facts and functional potential of quinoa (Chenopodium quinoa Willd.), an ancient Andean grain: a review. J Sci Food Agric. 2010; 90: 2541-2547.

4. Lutz M, Bascuñán-Godoy L. The revival of quinoa: a crop for health. In: Superfood and Functional Food - An Overview and its Utilization to Processed Foods (V. Waisundara, M. Shiomi, Eds.) In Tech Open. 2017, p 37-54.

5. Fuentes F, Maughan PJ, Jellen ER. Genetic diversity and genetic resources for the improvement of quinoa (Chenopodium quinoa Willd.). Rev Geogr Valpso. 2009; 42: 20-33.

6. Martínez $E A$, Veas $E$, Jorquera $C$, San Martín $R$, Jara $P$. Re-introduction of quinoa into Arid Chile: Cultivation of two lowland races under extremely low irrigation. J Agron Crop Sci. 2009; 195: 1-10.

7. Fuentes F, Bazile D, Bhargava A, Martínez EA. Implications of farmers' seed exchanges for on-farm conservation of quinoa, as revealed by its genetic diversity in Chile. I Agric Sci. 2012; 150: 702-716.

8. Bazile D, Jacobsen, SE, Verniau A. The global expansion of quinoa: trends and limits. Front Plant Sci. 2016; 7: 622.

9. Codex Alimentarius Commission. Request for comments at step 8 on the draft standard for quinoa. CL 2018/68CPL, Rome, 2018, p 3-5.

10. FAO. Quinoa: an ancient crop to contribute to world food security, 2011. Available in: http://www.fao.org/3/ aq287s/aq287s.pdf

11. Martínez EA, Maureira H, Miranda M, Quispe I, Rodríguez $M$, Vega A. Nutritional aspects of six quinoa (Chenopodium quinoa Willd.) ecotypes from three geographical areas of Chile. Chilean J Agric Res 2012; 72: 175-82.

12. Pefaur J. Quinoa in Chile, the take off an ancestral grain. Quinoa, potential development, public-private alliance, challenges for The World Congress. Office of Agriculture Studies and Policies (ODEPA), 2018. Available in: http:// www.odepa.gob.cl/wp-content/uploads/2018/02/ quinoa_final2018.pdf

13. FAO/WHO. Procedural Manual of the Codex Alimentarius Commission 26th edition Rome, 2018. Available in: http:// www.fao.org/documents/card/es/c/I8608EN/.

14. FAOWHO. Platform of information on quinoa, 2018. Available in: http://www.fao.org/in-action/quinoa-platform/ en/

15. Codex Alimentarius. International Food Standards. Standard for quinua - CXS 333-2019. FAO/WHO, Ed., Rome, Italy, 2019.

16. Bravo F, Espinoza C, Ganoza L, Gómez I, Reyes M. Peruvian Food Composition Tables. National Center of Foods and Nutrition, National Institute of Health. Lima, Perú. 2009, p 16-17.

17. Repo-Carrasco Valencia R, Serna LA. Quinoa (Chenopodium quinoa Willd.) as a source of dietary fiber and other functional components. Ciencia Tecnol Alim. 2011; 31: 225-30.

18. Koziol M. Chemical composition and nutritional evaluation of quinoa (Chenopodium quinoa Willd). J Food Comp Anal. 1992; 5: 35-68.

19. Schmidt-Hebbel H, Pennacchiotti I, Masson L, Mella MA. Chemical Composition Tables of Chilean Foods. 8th Ed, Universidad de Chile, Santiago, 1992, p. 16.

20. Repo-Carrasco R, Espinoza C, Jacobsen SE. Nutritional value and use of the Andean crops quinoa (Chenopodium quinoa) and kañiwa (Chenopodium pallidicaule). Food Rev Int. 2003; 19: 179-189.

21. Ogungbenle NH. Nutritional evaluation and functional properties of quinoa (Chenopodium quinoa) flour. Int J Food Sci Nutr. 2003; 54: 153-158.

22. Ando $H$, Chen YC, Tang H, Shimizu M, Watanabe $K$, Mitsunaga $Y$. Food components in fractions of quinoa seed. Food Sci Technol Res. 2002; 8: 80-84.

23. Abugoch LE. Quinoa (Chenopodium quinoa Willd.): Composition, chemistry, nutritional and functional properties. Adv Food Nutr Res. 2009; 58: 1-31.

24. Gonzalez JA, Konishi Y, Bruno M, Valoy M, Prado FE. Interrelationships among seed yield, total protein and amino acid composition of ten quinoa (Chenopodium quinoa) cultivars from two different agroecological regions. J Sci Food Agric. 2012; 92: 1222-1229.

25. Vidueiros SM, Curti RN, Dyner LM, Binaghi MJ, Peterson $G$, Bertero HD, et al. Diversity and interrelationships in nutritional traits in cultivated quinoa (Chenopodium quinoa Willd.) from Northwest Argentina. I Cereal Sci. 2015; 62: 87-93.

26. Nowak V, Du J, Charrondiere UR. Assessment of the nutritional composition of quinoa (Chenopodium quinoa Willd.) Food Chem. 2016; 193; 47-54.

27. USDA. Quinoa (Chenopodium quinoa Willd). National Nutrient Database Basic Report: 20035, Quinoa, uncooked. 2018. Basic Report 20137, Quinoa, cooked. 2018.

28. Miranda M, Vega-Gálvez A, Uribe G, López J, Martínez AE, Rodríguez MJ et al. Physico-chemical analysis, antioxidant capacity and vitamins of six ecotypes of Chilean quinoa (Chenopodium quinoa Willd). Proc Food Sci. 2011; 1: 1439-1446.

29. Alarcón $M$, Bustos $M$, Mendez D, Fuentes E, Palomo I, Lutz M. Effects of quinoa (Chenopodium quinoa Willd.) and lupin beans (Lupinus spp.) extracts on human platelet aggregation. Plant Foods Hum Nutr 2020; 75: 215-222.

30. Vega-Gálvez A, Zura L, Lutz M, Jagus R, Agüero M, Pastén $A$, et al. Assessment of dietary fiber, isoflavones and phenolic compounds with antioxidant and antimicrobial properties of quinoa (Chenopodium quinoa Willd.). Chilean J Agric Sci. 2018; 34: 57-67.

31. Fuentes F, Figueroa C, Olguín P, Duarte L, Ojeda M, Martínez E, et al. Competitive Potential of Chilean Quinoa. Santiago de Chile: Agricultural Innovation Foundation, Santiago, Chile. 2017, p 89-98.

32. Torrez M, Carvajal R, Guzmán A. Nutritional value of 10 varieties of quinoa (Chenopodium quinoa Willd) from Bolivian Altiplane. BIOFARBO. La Paz, Bolivia. 2002, p 55-60. 